\title{
TOOL MAGAZINE ARRANGEMENT AND OPERATIONS SEQUENCING ON CNC MACHINES
}

\author{
Selcuk Avcił and M. Selim Akturk†§ \\ Department of Industrial Engineering, Bilkent University, 06533 Bilkent Ankara, Turkey
}

(Received December 1994; in revised form February 1996)

\begin{abstract}
Scope and Purpose-An effective tool management is a major requirement for the implementation of a flexible manufacturing system (FMS), hence the computer numerical control (CNC) machine tools. A new solution methodology to the tool management problem is proposed in connection with the tool magazine arrangement and operations sequencing decisions, where there can be alternative tools for each operation. The proposed approach not only improves the overall solution in terms of the total manufacturing cost, but also prevents any infeasibility that might occur at the system level due to tool management constraints through a feedback mechanism.
\end{abstract}

\begin{abstract}
A two-phase approach is developed to solve the tool magazine arrangement and operations sequencing problems. The overall aim is to minimize the total manufacturing cost by utilizing the benefits of tool sharing concept and loading duplicate tools due to a possible decrease in tooling and tool operating costs while maintaining the feasibility in terms of precedence, tool magazine capacity, tool life covering and tool availability constraints due to tool contention among the operations for a limited number of tool types, because the absence of such crucial constraints may lead to infeasible results. Furthermore, the proposed approach can provide an effective decision making tool for the short term operational decisions of FMS. Copyright (C) 1996 Elsevier Science Ltd
\end{abstract}

\section{NOMENCLATURE}

$\alpha_{j}, \beta_{j}, \gamma_{j}$

$a_{i i^{\prime} k}$ :

$a_{j}:$

$\mathrm{CO}$ :

$C_{j p}$ :

$C_{t_{j}}:$

$D_{i}$ :

$d_{i}$ :

$f_{i j}$ :

I:

$\bar{I}_{j}:$

$J:$

J:

J:

$J_{i:}$ :

$L_{j}:$

$N_{b}$ :

$N_{m}$ :

$n_{\mathrm{ij}}$ :

$P_{j}$ :

$R L_{i, i^{\prime}}$ :

$T C_{j}$ :

$T_{i j}:$
Speed, feed, depth of cut exponents for the tool $j$

$0-1$ binary variable which is equal to 1 if the operations $i$ and $i^{\prime}$ are sharing the same tool type $j$ and $k^{\text {th }}$ duplicate of the requirement level $l$

The number of tool slots allocated for the tool type $j$

Operating cost of the CNC machine, $(\$ / \mathrm{min})$

Total cost of the alternative arrangement $p$ for the tool type $j$

Cost of the tool $j$, (\$/per tool)

Diameter of the generated surface, (in.)

Depth of cut for the operation $i$, (in.)

Feed rate for the operation $i$ using the tool type $j$, (ipr)

Set of all operations

Set of operations assigned to the tool $j$

Set of the available tools

Set of the allocated tools

Set of the arranged tools

Set of the candidate tools for the operation $i$

Set of possible tool requirement levels

Batch size

The capacity of the tool magazine

Number of tools required for the machining of volume $i$ by the tool $j$

A set of feasible number of tool slots that can be allocated to the tool type $j$

A set of feasible tool requirement levels for a pair of the operations $\left(i, i^{\prime}\right)$

Taylor's tool life constant for the tool $j$

Tool life of the tool $j$ for the operation $i$, (min)

$\dagger$ To whom all correspondence should be addressed (email: akturk@bilkent.edu.tr).

† Selcuk Avci is a research assistant in the Department of Industrial Engineering at Bilkent University, Turkey. He holds a B.S.M.E. from Middle East Technical University (METU), Turkey and a M.S.I.E. from Bilkent University, Turkey. His current research interests include hierarchical planning, machining economics and optimization theory. He is a student member of INFORMS.

$\S \mathrm{M}$. Selim Akturk is Assistant Professor of Industrial Engineering at Bilkent University, Turkey. He holds a Ph.D. in Industrial Engineering from Lehigh University, U.S.A., and B.S.I.E. and M.S.I.E. from Middle East Technical University (METU), Turkey. His current research interests include hierarchical planning and control of large scale systems, cellular manufacturing systems, and advanced manufacturing technologies. Dr. Akturk is a senior member of IIE and member of INFORMS. 


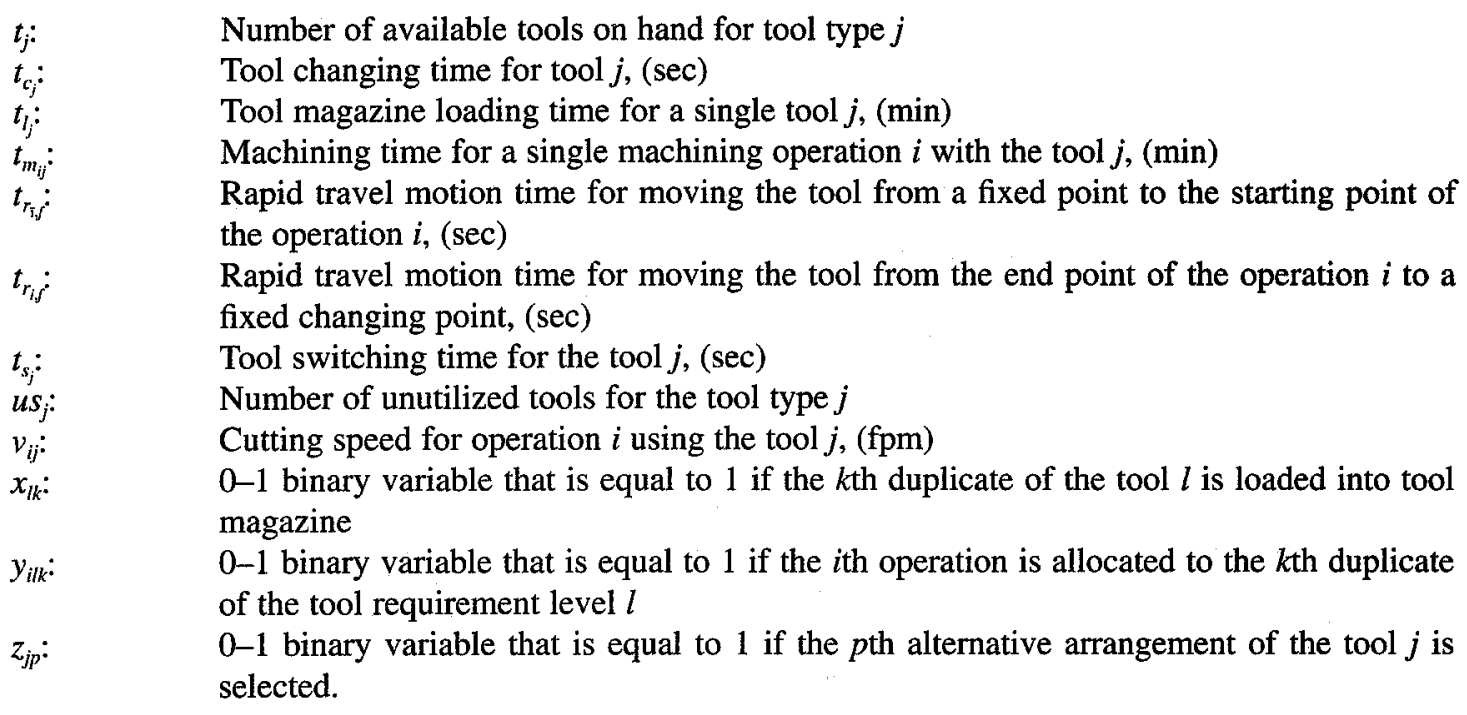

\section{INTRODUCTION}

The tool management concept has become an important issue for the successful operation and the effective utilization of the automated manufacturing systems in both system and the machine levels due to its impact on the total manufacturing cost as stated by Agapiou [1] and Kouvelis [8]. Gray et al. [6] give an extensive survey on the tool management issues on automated manufacturing systems, and emphasize the fact that the lack of tooling considerations has resulted in poor performance of these systems. They also present an integrated conceptual framework for resource planning to examine how tool management issues can be classified into tool-level, machine-level, and system-level concerns. In a previous study by Akturk and Avci [2] we address the issues at the tool level including the determination of optimum machining conditions and tool allocation. In this study, we discuss machine-level issues related to tooling a single $\mathrm{CNC}$ machine.

Kiran and Krason [7] discuss tool management issues in detail as a vital part of a flexible manufacturing system (FMS) and observe that tooling requirements generally exceed the tool magazine capacities in most practical FMSs. In this research, tooling decisions are limited to the cutting tools residing in an CNC tool magazine as opposed to jigs and fixtures that may be used to support the machining operation. In the FMS literature, operational problems at the machine level concerning tool magazine arrangement and operations sequencing decisions have been rarely addressed. However, the operational problems, such as tool sharing, tool availability, loading duplicate tools, precedence, tool magazine capacity and tool life, should be taken into account for a reliable modeling of FMSs, otherwise the absence of such crucial constraints may lead to infeasible results. Furthermore, an inclusion of these issues in the process planning will provide an effective decision making tool for the short term operational decisions of FMS as discussed by Suri and Whitney [11].

The tool management problem has been studied by Bard [4], Tang and Denardo [12], Crama et al. [5] and Sodhi et al. [10], to minimize the number of tool switches due to a change in part mix. These studies assume constant processing times and tool lives, even though the tool wear can have a significant impact on the tool replacement frequency as stated by Gray et al. [6] and Sarin and Chen [9]. Furthermore, almost all of the studies in the literature consider the tool management and tool magazine arrangement problems at the system-level in an aggregated manner by defining $0-1$ binary variables to indicate that a particular tool $j$ is assigned to operation $i$. They, consequently, ignore a possibility of tool sharing and loading duplicate tools due to tool contention among the operations for a limited number of tool types as a result of the tool availability and tool life limitations. Tool sharing can also decrease both the tooling requirements and the non-machining time components, hence the total production cost. The problem of tool allocation and tool scheduling for a FMS is addressed by Amoako-Gyampah et al. [3]. Four tool allocation and three tool scheduling strategies are compared at the system-level through a simulation study. They show that the use of different tooling strategies produces significantly different outcomes in FMSs performance. We propose a new solution methodology to solve for the tool magazine arrangement and operations sequencing problems simultaneously by allowing more accurate portrayal of the operation 
of CNC machines with an inclusion of tool contention, tool life, precedence and tool magazine capacity restrictions.

\section{PROBLEM DEFINITION}

In a previous study by Akturk and Avci [2] the tooling issues related to the machining conditions, tool availability and tool wear restrictions are addressed at the tool-level. A new solution methodology is developed to determine the optimum machining conditions and tool allocation simultaneously for a multiple operation case where there can be alternative tools for each operation. The constraints and the decision variables for machining conditions, tool and operation assignments, and operations sequence interact with each other as shown in Fig. 1, which illustrates the proposed decision hierarchy for

Sets of Machinable Volumes and Candidate Tools

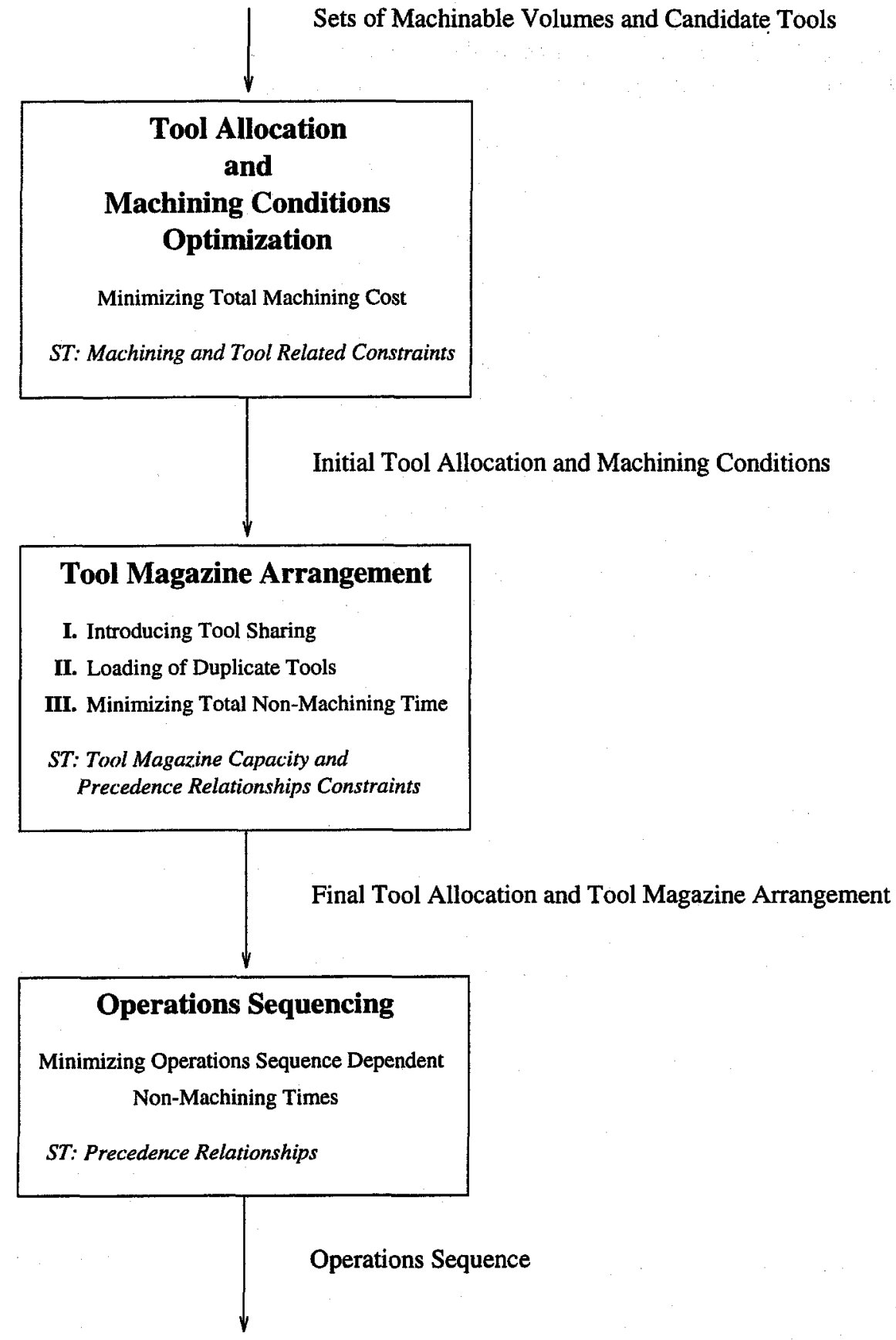

Fig. 1. Proposed decision hierarchy. 
machining conditions optimization, tool magazine arrangement and operations sequencing problems for a CNC machine. As indicated in the block diagram, the machine-level issues are relaxed in the previous study.

In this study, we emphasize the tool management issues at the CNC machine level. We extend the previous results by including the tool magazine arrangement and the operations sequencing problems in connection with the precedence, tool life covering and tool magazine capacity constraints to manufacture a batch of parts on a CNC machine. The objective criterion is minimizing the total manufacturing cost that counts for both operating cost due to non-machining events and tooling cost. All time consuming events except the actual cutting operation are called non-machining time components. The following oneś should be minimized since they are directly affected by the tool management and operations sequencing decisions:

- Tool Switching Time: Is the time required to replace the worn tool with a new one; each tool type might have a different switching time.

- Rapid Travel Motion Time: Is the time needed to relocate the tool from one point to another, e.g. from tool magazine to starting point of the cutting operation, which can be expressed as follows:

$$
t_{s_{x y y}}= \begin{cases}2 \sqrt{\frac{\Delta_{x, y}}{t s a}} & , \text { if } \Delta_{x, y} \leq \frac{t s s^{2}}{2 t s a} \\ \frac{\Delta_{x, y}}{t s s}+\frac{t s s}{t s a} & , \text { if } \Delta_{x, y} \frac{t s s^{2}}{2 t s a}\end{cases}
$$

where $t s s$ and $t s a$ are the speed and acceleration of the machine tool slides, respectively, and $\Delta_{x, y}$ is the shortest linear distance between the points $x$ and $y$.

- Tool Interchanging Time: Is the time necessary to move a tool from the tool holder to the tool magazine and replace it back, or vice versa.

Although there may be other distinct non-machining time components such as tool tuning, spindle acceleration/deceleration, workpiece loading/unloading, etc., we consider only the ones that can be expressed either as a function of tool magazine arrangement or operations sequence, or those that can vary between the different tool and operation pairs.

In this study, we define the machining time to tool life ratio as the tool usage rate, denoted by $U_{i j}$. In particular, the following expression is derived for the turning operation, and a similar expression can be derived for other operations:

$$
U_{i j}=\frac{t_{m_{i j}}}{T_{i j}}=\frac{\pi \cdot D_{i} \cdot L_{i} \cdot d_{i}^{\gamma_{j}}}{12 \cdot T C_{j} \cdot v_{i j}^{\left(1-\alpha_{j}\right)} \cdot f_{i j}^{\left(1-\beta_{j}\right)}}
$$

The following assumptions are made to define the scope of the study:

- There is a tool magazine, equipped with automatic tool-changers, attached on each CNC machine with a limited tool slot capacity.

- Only a single tool can be switched at any time.

- Each tool may fit in any slot of the tool magazine and we do not deal with the tool magazine balancing problem.

- There exists a limited quantity for each tool type, and the optimum machining conditions and tool usage rates are already found in Akturk and Avci. [2]

\section{TOOL MAGAZINE ARRANGEMENT}

The tools needed for the manufacturing of a batch of parts should be assigned to operations individually and placed into the tool magazine by considering the available quantities on hand and the capacity of the magazine. For any tool type, loading duplicate tools into the tool magazine might be necessary to satisfy the tool life covering constraint because of the given machining conditions. On the other hand, we may want to assign more than one operation to a certain tool to introduce tool sharing among the operations. This may provide a potential gain by reducing non-machining times hence the manufacturing cost. Furthermore, tool sharing can prevent potential infeasibilities that might incur for the 
tool magazine capacity constraint due to the initial tool loading. The following algorithm is proposed to find the best tool magazine arrangement by considering both tool sharing events and tool duplicates. In this algorithm, we identify any tool duplicate by its tool type $j$ and the requirement level $l$, which is defined as the number of tools needed to complete a set of operations in manufacturing a batch of parts.

The steps of the proposed algorithm can be summarized as follows:

Step 1: Allocate single operation tools

Step 2: Determine tool requirements for duplicates

Step 3: Find the minimum tool slot requirements for each tool

Step 4: Check the tool magazine capacity constraint

Step 5: Generate a set of feasible tool slot requirements

Step 6: Develop allocations to slots to minimize interchange requirements

Step 7: Evaluate cost of each assignment

Steps 8 and 9: Select an optimal strategy based on the reduced feasible set.

Furthermore, a step-by-step illustration of the proposed algorithm is given in Section 6 on an example part.

- Step 1: For the tool types having only a single operation assignment, a single slot should be allocated in the tool magazine, such that $a_{j}=1$ and $j \in \breve{J}$.

- Step 2: For the tools having more than one operation in their assignment set, determine the possible tool requirement levels, $l \in L_{j}$, and the maximum allowable tool usage rate, $M_{l}$, that can be assigned for a set of operations, respectively:

$$
L_{j}=\left\{l l l \in\left\{\min _{i \in \bar{I}_{j}}\left\{n_{i j}\right\}, \ldots,\left[N_{b} \backslash\left[\frac{N_{b}}{t_{j}}\right]\right]\right\} \forall l, l^{\prime} \in L_{j}, l \neq l^{\prime}, M_{l} \neq M_{l^{\prime}}\right\}
$$

and $M_{l}=1 /\left[\frac{N_{b}}{l}\right]$.

Also create a set of operations which can be performed at this level such that $V_{l}=\left\{i \mid i \in \bar{I}_{j}\right.$ and $\left.U_{i j} \leq M_{l}\right\}$ for every $l \in L_{j}$, and generate the index set for the duplicates of this requirement level such that $K_{l}=\left\{1,2, \ldots, \min \left\{s\left(V_{l}\right),\left[t_{j} / l\right]\right\}\right\}$.

- Step 3: For every tool $j$ in set $\bar{N}$, the following 0-1 IP model is solved to find the minimum tool slot requirement in the tool magazine while maintaining a feasible allocation of the operations of tool type $j$ :

$$
\begin{aligned}
& \text { Minimize } \epsilon_{j}=\sum_{l \in L_{j}} \sum_{k \in K_{l}} x_{l k} \\
& \text { Subject to } \sum_{i \in V_{l}} U_{i j} \cdot y_{i k} \leq M_{l} \cdot x_{l k}, \forall l \in L_{j} \text { and } k \in K_{l} \\
& \sum_{l \in L_{j}} \sum_{k \in K_{l}} y_{i l k}=1, \forall i \in V_{l} \\
& \sum_{l \in L, j} \sum_{k \in K_{l}} l \cdot x_{l k} \leq t_{j} \\
& \sum_{l \in L_{j}} \sum_{k \in K_{l}} x_{l k} \leq N_{m}-\sum_{j \in J} a_{j}-((s \bar{\Lambda} \breve{J})-1)
\end{aligned}
$$

The first constraint ensures that the maximum allowable tool usage rate will not be exceeded, and if an operation is assigned to a particular tool requirement level then this tool will be allocated in the tool magazine. The second constraint ensures that every operation is assigned to a single tool. The third constraint avoids exceeding the number of available tools. Finally, the last constraint represents the upper bound on the available number of tool slots for each tool, since loading duplicate tools will require additional slots in the tool magazine.

- Step 4: Check the tool magazine capacity constraint as follows:

$$
\sum_{j \in \lambda_{j}^{j}} \epsilon_{j}+\sum_{j \in J} a_{j} \leq N_{m}
$$




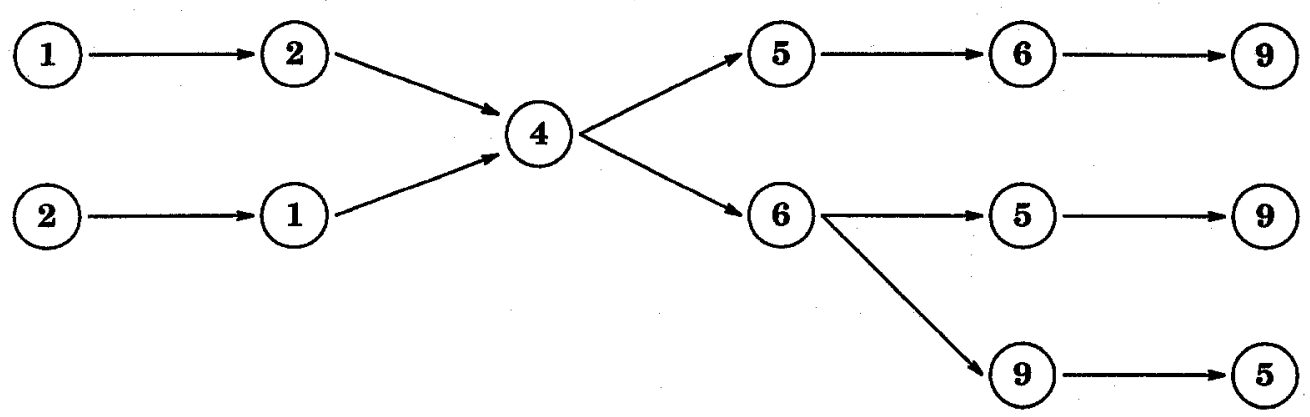

Fig. 4. Possible orderings of the operations of tool 1.

If the above constraint is violated, then the problem is infeasible and one should either decrease tool usage rates by changing the machining conditions, or re-arrange the tool-operation assignments.

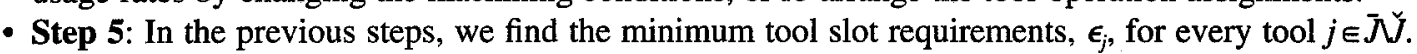
Furthermore the maximum number of tool slots that can be assigned, $\sigma_{j}$, is the minimum of the following three numbers: number of tool slots available after allocating minimum number of slots for the other tool types, number of tools available on hand for this tool type, i.e. allocating a separate tool slot for every tool of this type, and number of operations assigned on this tool type. That is $\sigma_{j}=\min \left\{\left(N_{m}-\Sigma_{i \in\left(\bar{N}_{j) y} \epsilon_{i}\right.}-\sum_{i \in j} a_{i}\right), t_{j}, s\left(\bar{I}_{j}\right)\right\}$. Therefore, we will search a set of possible tool slot requirements, $p \in P_{j}$, such that $P_{j}=\left\{\epsilon_{j}, \epsilon_{j}+1, \ldots, \sigma_{j}\right\}$. In the following steps, we will find the best tool magazine arrangement, $p \in P_{j}$ for every tool $j \in \overline{\mathcal{N}}$.

- Step 6: For every tool $j \in \bar{N} \tilde{J}$, build a precedence relationship graph to illustrate the possible orderings of the operations assigned on the tool type $j$. An example of such a graph is given in Section 6, Fig. 4. Create a set of operation pairs that can be machined adjacently due to the precedence relations such that $A_{j}=\left\{\left(i, i^{\prime}\right) l i, i^{\prime} \in \bar{I}_{j}, i \neq i^{\prime}\right\}$ and a set of possible tool requirement levels, $R L_{i, i^{\prime}}$, for every pair $\left(i, i^{\prime}\right)$ such that $R L_{i, i^{\prime}}=\left\{l \mid U_{i j}+U_{i^{\prime} j} \leq M_{i}\right\}$. By using these sets, a $0-1$ IP model is developed to find the best tool magazine arrangement of tool $j$ for the given number of tool slots, $p \in P_{j}$, which results in a minimum number of tool interchanging events and corresponds to the best tool sharing combination. The primary criterion is to increase the number of tool sharing events due to its potential benefits in decreasing the non-machining operation costs like tool interchanging and switching, while minimizing the total tool requirements for a given tool sharing combination to decrease the tooling cost as a secondary criterion. This will minimize the number of times tool magazine reloading occurs. Therefore, a preemptive priority scheme is applied by taking $\rho_{1} \gg \rho_{2}$ in the objective function for a maximization problem.

Maximize $O_{j p}=\rho_{1} \cdot\left(\sum_{t \in L_{j}} \sum_{k \in K_{l}} \sum_{\left(i, i^{\prime}\right) \in A_{j}} a_{i i^{\prime} \prime k}\right)+\rho_{2} \cdot u s_{j}$

Subject to: $\sum_{i \in V_{l}} U_{i j} \cdot y_{i l k} \leq M_{l} \cdot x_{l k}, \forall l \in L_{j}$ and $k \in K_{l}$

$$
\begin{aligned}
& \sum_{l \in L_{j}} \sum_{k \in K_{l}} y_{i l k}=1, \forall i \in V_{l} \\
& \sum_{l \in L_{j}} \sum_{k \in K_{l}} l \cdot x_{l k}+u s_{j}=t_{j} \\
& \sum_{l \in L_{j}} \sum_{k \in K_{l}} x_{l k}=p \\
& 2 a_{i i^{\prime} \prime k} \leq y_{i l k}+y_{i^{\prime} k}, \forall\left(i, i^{\prime}\right) \in A_{j}, l \in R L_{i, i^{\prime}} \text { and } k \in K_{l}
\end{aligned}
$$

In this formulation, $a_{i i^{\prime} k}$ is a $0-1$ binary variable which is equal to 1 if operations $i$ and $i^{\prime}$ are sharing the same tool type $j$ at the $k$ th duplicate of the requirement level $l$, and $u s_{j}$ counts for the number of unused tools.

- Step 7: Evaluate the following cost measure for every alternative arrangement of tool $j \in \bar{N} \breve{J}$, where 
$A S_{l k}=\left\{\left(i, i^{\prime}\right) \in A_{j} \mid y_{i l k}=y_{i^{\prime} k}=1\right\}$, is the set of adjacent operations assigned on the same tool, i.e. the $k$ th duplicate of the $l$ th requirement level of tool type $j$, and $D T_{l k}=\left\{i \in \bar{I}_{j} \mid y_{i k}=1\right\}$, is the set of distinct operations assigned on the same tool:

$$
\begin{aligned}
& C_{j p}=C O \cdot N_{b} \cdot \sum_{i \in L_{j}} \sum_{k \in K_{i}}\left[\left[\sum_{\left(i, i^{\prime}\right) \in A S_{|l|}} t_{r_{i, i}}\right)+t_{r_{j, i}}+t_{r_{i}^{\prime}, f^{\prime}}+2 \cdot t_{c_{j}}\right] \\
& \left.+\sum_{i \in\left(D T_{l k} \backslash A s_{j k}\right)}\left(t_{r_{r} \mathrm{~T}}+t_{r_{i j}}+2 \cdot t_{c_{j}}\right)\right]+C O \cdot\left[\left(t_{j}-u s_{j}\right)-a_{j}\right] \cdot t_{s_{j}} \\
& +\left[C O \cdot a_{j} \cdot t_{i}\right]+C_{t_{j}} \cdot\left(t_{j}-u s_{j}\right)
\end{aligned}
$$

In the first term, the rapid travel motion (RTM) and the tool interchanging times are found by considering the tool sharing information which is embedded in set $A S_{l k}$ including a chain of adjacent operations which satisfy the precedence relationships. The second term also accounts for the RTM and tool interchanging times of the distinct operations excluded by set $A S_{l k^{*}}$. The other terms represent the tool switching, loading and tooling costs, respectively.

- Step 8: For every tool in set $\bar{N}$ determine the tool slot requirement which results in minimum cost such that $\mu_{j}=\arg \min _{p \in P_{j}}\left\{C_{j p}\right\}$. Therefore, for every tool type $j$, we have the best number of tool slot requirement, $\mu_{j}$, that gives the minimum cost. In addition, we have already allocated tool slots, $a_{j}$, for every $j \in \tilde{J}$. Consequently, we check the tool magazine capacity constraint as follows:

$$
\sum_{j \in \mathcal{N} J} \mu_{j}+\sum_{j \in J} a_{j} \leq N_{m}
$$

If the above condition is violated then go to Step 9. Otherwise, stop and the best arrangements are the ones resulting in minimum costs.

- Step 9: Determine the best tool arrangement of every tool type in $\check{N}$ by solving the following 0-1 $\mathrm{IP}$, where $z_{j p}$ is a $0-1$ binary variable that is equal to 1 if the $p$ th alternative arrangement of tool $j$ is selected:

$$
\begin{aligned}
\text { Minimize } & \sum_{j \in \bar{N}} \sum_{p \in P_{J}} z_{j p} \cdot C_{j p} \\
\text { Subject to: } & \sum_{p \in P_{j}} z_{j p}=1, \forall j \in \bar{J} \breve{J} \\
& \sum_{j \in \bar{N}} \sum_{p \in P_{j}} z_{j p} \cdot a_{j} \leqslant N_{m}-\sum_{j \in \breve{J}} a_{j}
\end{aligned}
$$

In this formulation, the first constraint requires that only one of the alternatives will be selected and the second constraint ensures that total tool slot requirement of the entire tool magazine arrangement will not exceed the tool magazine capacity.

In this algorithm, we utilize a two-phase approach. In the first phase, we apply a pre-processing on the input data to reduce the solution space and find an initial feasible solution. Then in the second phase, we search for a better solution while maintaining the feasibility. In Step 1, the tools having only a single operation assignment are placed into the tool magazine, since there is no other alternative. Step 2 applies a pre-processing on the input data to reduce the search space by imposing some feasibility constraints. The minimal tool slot requirements are found for the remaining tools in Step 3 and the tool magazine capacity constraint is checked in Step 4 to verify the initial feasibility of the problem.

If the initial feasibility is verified then the alternative solutions are generated and evaluated in the second phase. For this purpose, in Steps 5 and 6 the possible tool slot requirements and the corresponding best tool-operation assignments are found, respectively. The minimum tool slot requirement also gives the lower bound on the possible tool slot requirements for every tool, whereas the upper bound is found as the minimum of the number of available tool slots, tools, and the cardinality of the operation assignment set. In Step 7, we propose a new cost measure to evaluate different tool arrangement alternatives, which include all the non-machining time components and the tooling cost since they are closely related with the tool magazine arrangement and tool sharing. Tool sharing information found at the previous step is used to determine partial information about the tool motions and to calculate the corresponding operating cost. Finally, the sum of the individual tool arrangements resulting in minimum costs gives the best solution, if it is feasible. Otherwise, the best solution is obtained in Step 9 by solving an IP model. 


\section{OPERATIONS SEQUENCING}

After fixing the operation and tool assignments and tool magazine arrangement, the operations sequencing decision remains to be made. Tool interchanging and rapid travel motion times are the only variables to be minimized at this level. This sequencing decision is transformed into a network model, in which nodes correspond to the several phases of a workpiece such as it is initially a raw material in State $o$, and every cutting operation changes the state of the workpiece. At the end, the final state having $m$ operations is denoted by the node $m+1$. Cutting operations are presented by the arcs and every arc will have a cost value corresponding to the sum of non-machining times due to state transitions. Furthermore, at each state, a set of operations, $S_{i}$, that can be done at state $i$, is defined by imposing the precedence relations between the operations. Our objective is to find the minimum path from root node $o$ to final node $m+1$. The following algorithm is proposed to make a full enumeration by spanning all feasible alternatives.

- Step 1: For the tools having a sequence of adjacent operations, define a new operation by taking the starting point of the first operation of this chain as the starting point, and ending point of the last operation as the ending point of aggregated volume.

- Step 2: Calculate the cost of arcs from root node $o$ to State 1 as follows:

$$
c_{o, i}=t_{c_{j}}+t_{r_{j, i}} \text {, where } i \in S_{1} \text { and } i \in \bar{I}_{j}
$$

- Step 3: For every intermediate State $n$, where $n \in\{1, \ldots, m-1\}$, calculate the cost of arc directed from State $S_{n}$ to $S_{n+1}$, where $i^{\prime} \in S_{n}, i \in S_{n+1}$, and $j^{\prime}$ and $j$ are their corresponding tools, respectively:

$$
c_{i^{\prime}, i}= \begin{cases}t_{r_{r^{\prime}, f}}+t_{c_{j^{\prime}}}+t_{c_{j}}+t_{f_{f, \mathrm{i}}} & , \text { if } j^{\prime} \neq j \\ t_{r_{i^{\prime}, \mathrm{s}}} & , \text { if } j^{\prime}=j\end{cases}
$$

- Step 4: Calculate the cost of arc from State $m$ to State $m+1$ as follows:

$$
c_{i, m+1}=t_{r_{i, j}}+t_{c_{j}}, \text { where } i \in S_{m}
$$

- Step 5: Calculate the total cost for every path from root node $o$ to leaf node $m+1$ and pick the minimum cost path as the operations sequence.

In the previous tool magazine arrangement algorithm, operations sharing the same tool are aggregated into a single virtual operation and a partial operation sequence is generated in Step 1. The operations sequencing problem for the remaining operations can be formulated as a traveling salesperson problem, which is proven to be NP-complete. If we relax the precedence relations between the operations, then the optimum operations sequence corresponds to the shortest Hamiltonian cycle starting from the fixed tool changing point, visiting every operation exactly once and returning back to the fixed point. Therefore we transform this sequencing decision into a directed graph by imposing the precedence relations as shown in Fig. 2. After finding the cost of each arc as discussed above, the problem is reduced to find the shortest path from root node $o$ to leaf node $m+1$ while performing exactly one operation at each state.

\section{A NUMERICAL EXAMPLE}

In this section, an example part, illustrated in Fig. 3, is studied which has twelve prespecified operations. The geometrical data and the machining conditions, that can be found at the first stage of the decision hierarchy presented in Fig. 1, are summarized in Table 1. There are four different tool types available whose data given in Table 2 . The following data characterize the operating conditions of the CNC machine tool.

- Operating cost, $C O: \$ 1 / \mathrm{min}$

- Speed of the slides, $t s s: 5 \mathrm{in} . / \mathrm{sec}$

- Acceleration of the slides, $t s a: 5 \mathrm{in} . / \mathrm{sec}^{2}$

- Tool magazine capacity, $N_{m}: 30$ slots

- Batch size, $N_{b}: 25$ workpieces

- Coordinates of the tool changing point, $f .(0,0,20)$ (in.). 


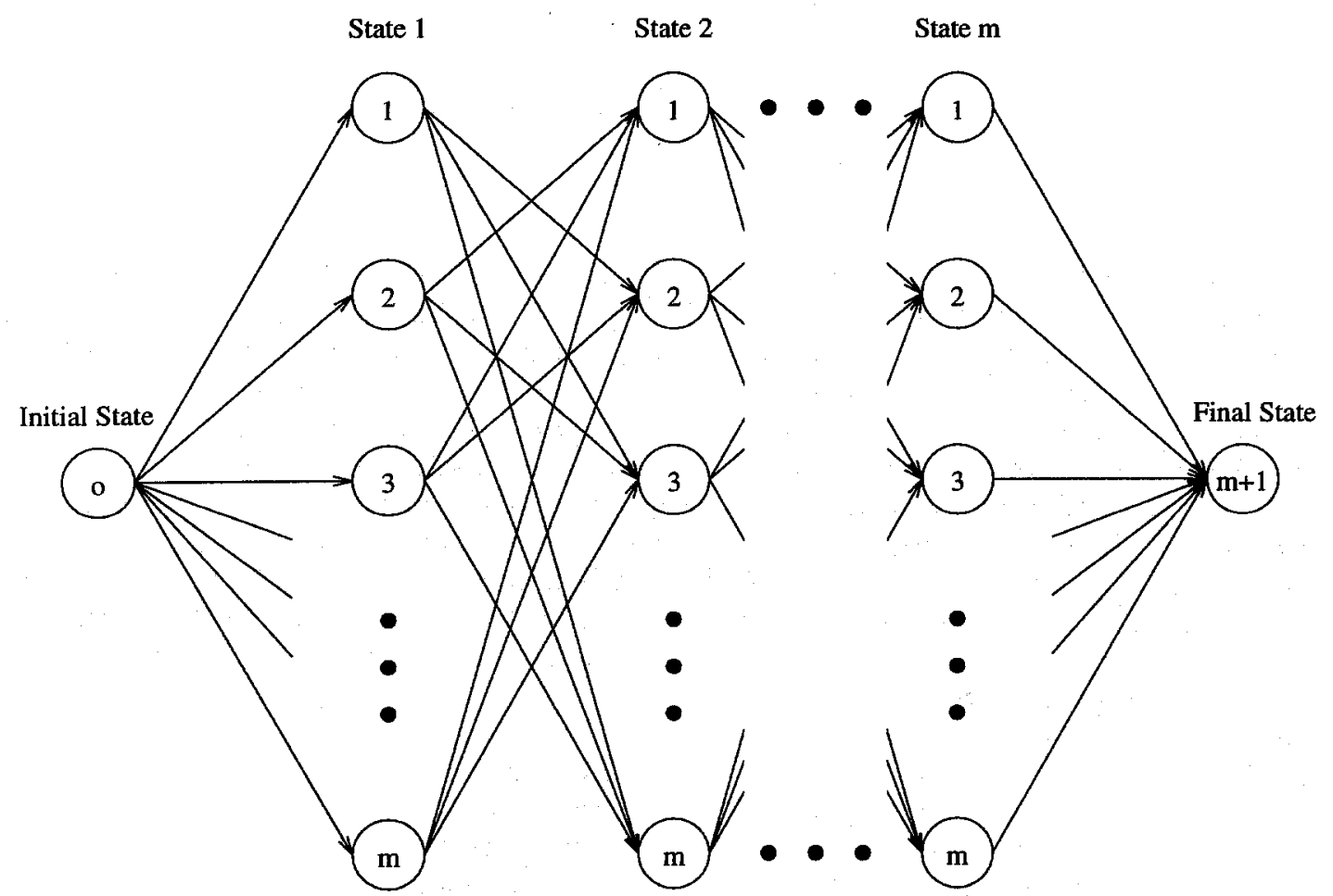

Fig. 2. The network model for operations sequencing problem.

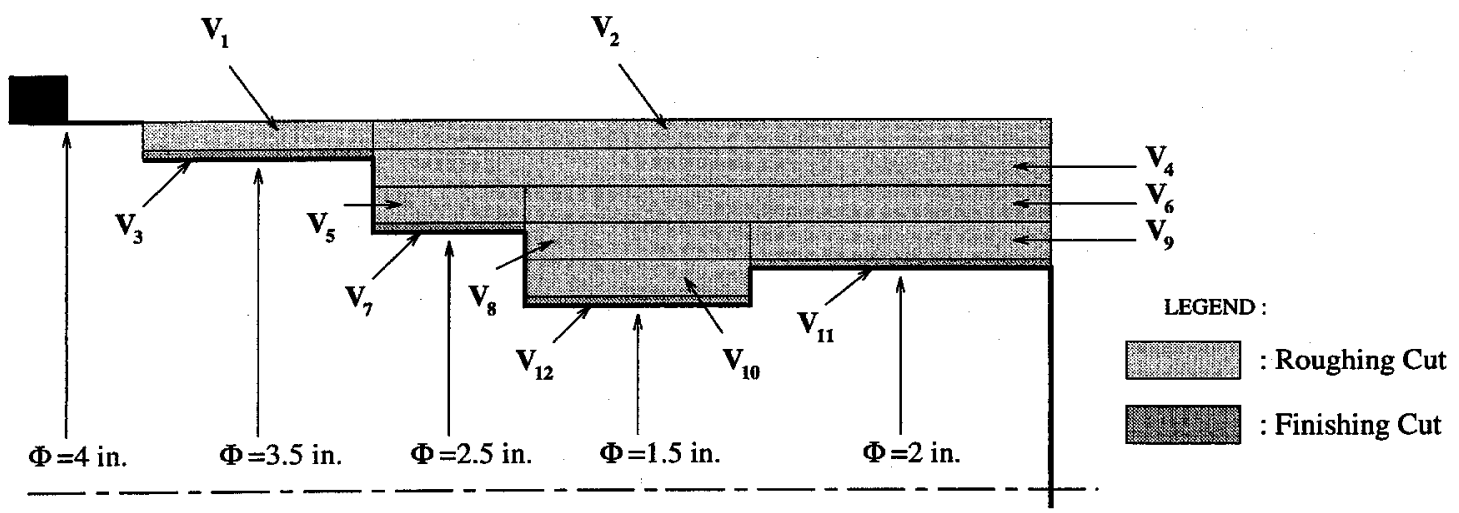

Fig. 3. Machinable volume presentation.

\begin{tabular}{lcccccccc}
\multicolumn{10}{c}{ Table 1. Machinable volume data, tool allocation and usages } \\
\hline$V_{\#} \#$ & $\mathrm{D}_{\mathrm{i}}$,(in.) & $\mathrm{L}_{\mathrm{i}}$ (in.) & $\mathrm{d}_{\mathrm{i}}$ (in.) & Start point & End point & $\mathrm{T} \#$ & $\mathrm{U}_{\mathrm{ij}}$ & $\mathrm{n}_{\mathrm{ij}}$ \\
\hline$V_{1}$ & 4 & 3 & 0.2 & $(4,2,0)$ & $(1,2,0)$ & 1 & 0.17 & 5 \\
$V_{2}$ & 4 & 9 & 0.2 & $(13,2,0)$ & $(4,2,0)$ & 1 & 0.45 & 13 \\
$V_{3}$ & 3.6 & 3 & 0.05 & $(4,1.8,0)$ & $(1,1.8,0)$ & 3 & 0.15 & 5 \\
$V_{4}$ & 3.6 & 9 & 0.25 & $(13,1.8,0)$ & $(4,1.8,0)$ & 1 & 0.36 & 13 \\
$V_{5}$ & 3.1 & 2 & 0.25 & $(6,1.55,0)$ & $(4,1.55,0)$ & 1 & 0.09 & 3 \\
$V_{6}$ & 3.1 & 7 & 0.25 & $(13,1.55,0)$ & $(6,1.55,0)$ & 1 & 0.26 & 9 \\
$V_{7}$ & 2.6 & 2 & 0.05 & $(6,1.3,0)$ & $(4,1.3,0)$ & 3 & 0.09 & 3 \\
$V_{8}$ & 2.6 & 3 & 0.25 & $(9,1.3,0)$ & $(6,1.3,0)$ & 2 & 0.24 & 7 \\
$V_{9}$ & 2.6 & 4 & 0.25 & $(13,1.3,0)$ & $(9,1.3,0)$ & 1 & 0.22 & 7 \\
$V_{10}$ & 2.1 & 3 & 0.25 & $(9,1.05,0)$ & $(6,1.05,0)$ & 3 & 0.16 & 5 \\
$V_{11}$ & 2.1 & 4 & 0.05 & $(13,1.05,0)$ & $(9,1.05,0)$ & 4 & 0.14 & 4 \\
$V_{12}$ & 1.6 & 3 & 0.05 & $(9,0.8,0)$ & $(6,0.8,0)$ & 4 & 0.11 & 3 \\
\hline
\end{tabular}




\begin{tabular}{lcccc}
\multicolumn{5}{c}{ Table 2. Tooling information } \\
\hline & $\mathrm{T}_{1}$ & $\mathrm{~T}_{2}$ & $\mathrm{~T}_{3}$ & $\mathrm{~T}_{4}$ \\
\hline$t_{s_{i}}(\mathrm{~min})$ & 0.5 & 0.75 & 1 & 0.75 \\
$t_{i_{j}}(\mathrm{~min})$ & 3 & 2 & 2.5 & 3 \\
$t_{c_{j}}(\mathrm{sec})$ & 5 & 5 & 5 & 5 \\
$t_{j}$ & 50 & 7 & 13 & 7 \\
$C_{t_{j}}$ & 2.50 & 2 & 1.75 & 2 \\
\hline
\end{tabular}

In Step 1, a single tool slot is allocated for tool 2 since it has only operation 8 in its assignment set. In Step 2, a pre-processing of the data for every tool type is performed to determine the possible tool requirement levels and their allowable tool usage rates, operation assignments and duplicates. This preprocessing is presented for tool 1 in Table 3, for example, at level 3 we can have a single duplicate tool replaced after producing 9 parts at the given usage rate, which restricts the set of operations that can share tool 1. Let us compare the initial tool allocation combinations available with the amount of reduction in the solution space after the proposed pre-processing step by imposing some feasibility constraints. As shown in Table 3, there are 5 feasible tool sharing possibilities and totally 7 alternatives of tool 1 with duplicate tool requirements after pre-processing as opposed to initial 63 tool allocation combinations even without tool duplicates. This corresponds to a more than $90 \%$ reduction in the solution space.

In Step 3, we evaluate the minimum tool slot requirement for every tool type by using an IP model. The following model is developed for tool 4:

Minimize $\epsilon_{4}=x_{3,1}+x_{4,1}+x_{5,1}+x_{7,1}$

Subject to:

$$
\begin{array}{ll}
0.14 y_{11,3,1} \leq 0.111 x_{3,1} & , \text { for the 1st duplicate of level } 3 \\
0.14 y_{11,4,1}+0.11 y_{12,4,1} \leq 0.143 x_{4,1} & \text {, for the 1st duplicate of level 4 } \\
0.14 y_{11,5,1}+0.11 y_{12.5,1} \leq 0.200 x_{5,1} & \text {, for the 1st duplicate of level 5 } \\
0.14 y_{11,7.1}+0.11 y_{12,7,1} \leq 0.250 x_{7.1} & \text {, for the 1st duplicate of level 7 } \\
y_{11,3,1}+y_{11.4,1}+y_{11,5.1}+y_{11,7,1}=1 & \text {, for the operation 11 } \\
y_{12,4,1}+y_{12,5,1}+y_{12.7,1}=1 & \text {, for the operation 12 } \\
3 x_{3,1}+4 x_{4,1}+5 x_{5,1}+7 x_{7,1} \leq 7 & \text {, tool availability } \\
x_{3,1}+x_{4,1}+x_{5,1}+x_{7,1} \leq 26 & \text {, tool magazine capacity. }
\end{array}
$$

The above mathematical model results in $\epsilon_{4}=1$, such that there exists a feasible tool-operation assignment of tool 4 which requires only a single slot in the tool magazine. Similarly, models for tools 1 and 3 give $\epsilon_{1}=2$ and $\epsilon_{3}=1$, respectively. In Step 4, the tool magazine capacity constraint is checked as follows:

$$
\epsilon_{1}+\epsilon_{3}+\epsilon_{4}+a_{2}=2+1+1+1=5 \leq N_{m}=30
$$

As a result, we can justify that there exists a feasible solution with the minimal slot allocations before proceeding with the search phase of the algorithm.

In Step 5, the following possible tool slot requirements created for each tool by using the minimal tool slot allocations and the other input data:

$$
\begin{gathered}
P_{1}=\{2, \cdots, \min \{27,50,6\}\}=\{2, \cdots, 6\} \\
P_{3}=\{1, \cdots, \min \{26,13,3\}\}=\{1,2,3\} \\
P_{4}=\{1, \cdots, \min \{26,7,2\}\}=\{1,2\}
\end{gathered}
$$

In the next step, we investigate the adjacent operation pairs of the same tool type that may result in tool sharing and enhance the manufacturing cost. The following operation pairs and their possible tool requirement levels are identified for each tool type by considering the possible orderings of the operations of the given part. These orderings are also illustrated for tool 1 in Fig. 4.

- For tool 1:

Table 3. Pre-processing of the data for tool 1

\begin{tabular}{lcccc}
\hline Tool req. $(l)$ & Duplicates $\left(s\left(K_{1}\right)\right)$ & \# of parts $\left(\left[N_{i} / l\right]\right)$ & Usage rate $\left(M_{i}\right)$ & Operations $\left(V_{i}\right)$ \\
\hline 3 & 1 & 9 & 0.111 & $\{5\}$ \\
4 & 1 & 7 & 0.143 & $\{5\}$ \\
5 & 2 & 5 & 0.200 & $\{1,5\}$ \\
7 & 3 & 4 & 0.250 & $\{1,5,9\}$ \\
9 & 4 & 3 & 0.333 & $\{1,5,6,9\}$ \\
13 & 3 & 2 & 0.500 & $\{1,2,4,5,6,9\}$ \\
25 & 2 & 1 & 1.000 & $\{1,2,4,5,6,9\}$ \\
\hline
\end{tabular}




$$
\begin{gathered}
A_{1}\{(1,2),(1,4),(2,4),(4,5),(4,6),(5,6),(5,9),(6,9)\} \\
R L_{1,2}=\{25\}, R L_{1,4}=\{25\}, R L_{2,4}=\{25\}, R L_{4,5}=\{13,25\}, R L_{4,6}=\{25\}, \\
R L_{5,6}=\{13,25\}, R L_{5,9}=\{9,13,25\}, R L_{6,9}=\{13,25\},
\end{gathered}
$$

- For tool 3:

$$
\begin{gathered}
A_{3}\{(3,7),(3,10),(7,10)\} \\
R L_{3,7}=\{7,9,13\}, R L_{3,10}=\{9,13\}, R L_{7,10}=\{7,9,13\}
\end{gathered}
$$

- For tool 4:

$$
\begin{gathered}
A_{4}=\{(11,12)\} \\
R L_{11,12}=\{7\}
\end{gathered}
$$

Now, an IP model is used in conjunction with the above data to span all alternative tool allocations for each tool type, such that solving this model for every tool slot requirement of set $P_{j}$ generates a feasible allocation of tool $j$. The following model is written for tool 4 and slot requirement of 1 , where weights $\rho_{1}$ and $\rho_{2}$ of the objective function terms are taken as 100 and 1 , respectively.

Maximize $O_{4,1}=100\left(a_{11,12,7,1}\right)+u s_{4}$

Subject to: $\quad 0.14 y_{11,3,1} \leq 0.111 x_{3,1}$

$$
\begin{aligned}
& 0.14 y_{11,4,1}+0.11 y_{12,4,1} \leq 0.143 x_{4,1} \\
& 0.14 y_{11,5,1}+0.11 y_{12,5,1} \leq 0.200 x_{5,1}
\end{aligned}
$$$$
0.14 y_{11,7,1}+0.11 y_{12,7,1} \leq 0.250 x_{7,1}
$$$$
y_{11,3,1}+y_{11,4,1}+y_{11,5,1}+y_{11,7,1}=1
$$$$
y_{12,4,1}+y_{12,5,1}+y_{12,7,1}=1
$$$$
3 x_{3,1}+4 x_{4,1}+5 x_{5,1}+7 x_{7,1}+u s_{4}=7
$$$$
x_{3,1}+x_{4,1}+x_{5,1}+x_{7,1}=1
$$$$
2 a_{11,12.7,1} \leq y_{11.7 .1}+y_{12,7,1}
$$

The solution for the above formulation is equal to $y_{11,7,1}=y_{12,7,1}=x_{7,1}=a_{11,12.7,1}=1$ and $u s_{4}=0$ with the objective function value $O_{4,1}=100$, which means operations 11 and 12 are sharing the same tool at the requirement level 7.

In Step 7, the proposed cost measure is evaluated for every alternative arrangement. The alternative arrangements of tool 1 corresponding to the slot requirements in $P_{1}$ are tabulated in Table 4 with their costs. These alternatives are also illustrated in Fig. 5 with the contribution of each cost term. In Table 4, the tool sharing events are indicated by the ordered sets of operations. For example, when we allocate two

\begin{tabular}{lcccc}
\multicolumn{5}{c}{ Table 4. Alternative arrangements of tool 1} \\
\hline Tool slot, $p$ & $l$ & $k$ & Operations & Cost \\
\hline 2 & 25 & 1 & $\{2,1\}$ & 184.83 \\
& 25 & 2 & $\{4,6,5,9\}$ & \\
3 & 5 & 1 & $\{1\}$ & 175.99 \\
& 13 & 1 & $\{2\}$ & \\
4 & 25 & 1 & $\{4,6,5,9\}$ & \\
& 3 & 1 & $\{5\}$ & 190.51 \\
& 7 & 1 & $\{9\}$ & \\
& 9 & 1 & $\{6\}$ & \\
& 25 & 1 & $\{2,4,1\}$ & \\
5 & 5 & 1 & $\{1\}$ & 211.12 \\
& 7 & 1 & $\{9\}$ & \\
& 9 & 1 & $\{6\}$ & \\
& 13 & 1 & $\{2\}$ & \\
6 & 13 & 2 & $\{4,5\}$ & \\
6 & 3 & 1 & $\{5\}$ & 231.8 \\
& 5 & 1 & $\{1\}$ & \\
& 7 & 1 & $\{9\}$ & \\
& 9 & 1 & $\{6\}$ & \\
& 13 & 1 & $\{3\}$ & \\
& 13 & 2 & $\{4\}$ & \\
\hline
\end{tabular}


tool slots for tool 1 , we will have two duplicates at the requirement level of 25 such as the second duplicate is shared by the operations $4,6,5$ and 9 in the given order. Therefore, these four operations are aggregated into a single operation, operation $4^{+}$, by preserving the operations sequence and the precedence relationships.

In Step 8, for the tools in set $\bar{\Lambda}$, the tool slot requirements corresponding to the minimum cost values are found as $\mu_{1}=3, \mu_{3}=2$ and $\mu_{4}=1$. Then, the following condition is shown to be satisfied:

$$
\mu_{1}+\mu_{3}+\mu_{4}+a_{2}=3+2+1+1=7 \leq N_{m}=30 .
$$

Therefore, the best tool magazine arrangement is summarized in Table 5.

In the next algorithm, the operations sequencing decision is made to minimize the total rapid travel motion and tool interchanging times. Initially, the number of operations is decreased due to the aggregate operations formed in the previous algorithm. For the search tree, the possible nodes and the arcs with the time values as their cost values are presented in Table 6 . The best operations sequence is found as $2 \rightarrow$ $1 \rightarrow 4^{+} \rightarrow 8 \rightarrow 10 \rightarrow 11^{+} \rightarrow 7^{+}$, where operations $11^{+}$and $7^{+}$are the new aggregate operations.

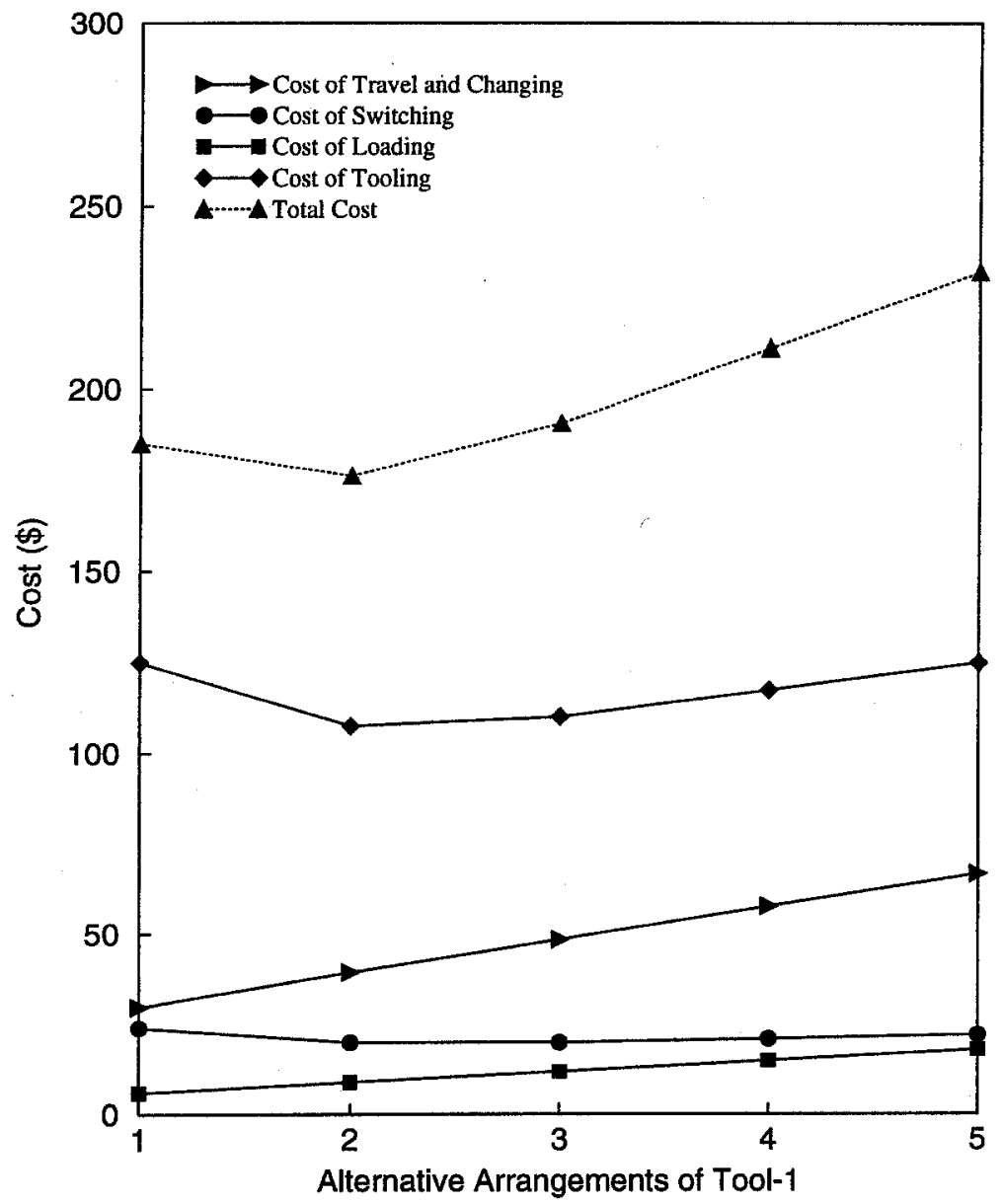

Fig. 5. Cost vs alternative arrangements of tool1.

Table 5. Final tool-operation assignments and tool magazine arrangement

\begin{tabular}{lccccc}
\hline Tool type & Duplicate & $l$ & $k$ & Operations & Cost \\
\hline 1 & 1st duplicate & 5 & 1 & $\{1\}$ & 175.99 \\
& 2nd duplicate & 13 & 1 & $\{2\}$ & \\
& 3rd duplicate & 25 & 1 & $\{4,6,5,9\}$ & \\
2 & Single & 7 & 1 & $\{8\}$ & 31.57 \\
3 & 1st duplicate & 5 & 1 & $\{10\}$ & 68.9 \\
& 2nd duplicate & 7 & 1 & $\{3\},\{7\}$ & \\
4 & Single & 7 & 1 & $\{11\},\{12\}$ & 35.56 \\
\hline
\end{tabular}


Table 6. Total times between the operations

\begin{tabular}{lcccccccccc}
\hline & $f$ & $V_{1}$ & $V_{2}$ & $V_{3}$ & $V_{4}^{+}$ & $V_{7}$ & $V_{8}$ & $V_{10}$ & $V_{11}$ & $V_{12}$ \\
\hline$f$ & $\propto$ & 8.10 & 8.79 & 8.10 & 8.78 & 8.18 & 8.39 & 8.39 & 8.78 & 8.39 \\
$V_{1}$ & 8.02 & $\infty$ & 6.40 & 4.60 & 6.40 & 5.01 & 5.61 & 5.61 & 6.41 & 5.62 \\
$V_{2}$ & 8.10 & 3.00 & $\infty$ & 3.40 & 5.80 & 4.30 & 5.01 & 5.02 & 5.81 & 5.03 \\
$V_{3}$ & 8.02 & 4.60 & 6.40 & $\infty$ & 6.40 & 5.00 & 5.60 & 5.61 & 6.40 & 5.61 \\
$V_{4}^{+}$ & 8.10 & 3.40 & 5.80 & 3.00 & $\infty$ & 4.28 & 5.00 & 5.01 & 5.81 & 5.02 \\
$V_{7}$ & 8.09 & 3.75 & 5.81 & 3.63 & 5.80 & $\infty$ & 5.00 & 5.00 & 5.80 & 5.00 \\
$V_{8}$ & 8.18 & 4.30 & 5.41 & 4.28 & 5.40 & 3.00 & $\infty$ & 4.60 & 5.40 & 4.61 \\
$V_{10}$ & 8.18 & 4.33 & 5.41 & 4.31 & 5.41 & 3.45 & 4.60 & $\infty$ & 5.40 & 4.60 \\
$V_{11}$ & 8.39 & 5.02 & 4.82 & 5.01 & 4.81 & 4.60 & 3.45 & 3.00 & $\infty$ & 3.45 \\
$V_{12}$ & 8.18 & 4.37 & 5.42 & 4.34 & 5.41 & 3.63 & 4.61 & 4.60 & 5.40 & $\infty$ \\
\hline
\end{tabular}

Therefore, the final operations sequence in detail, including the tool sharing events denoted in square brackets, is given in the below:

$$
2 \rightarrow 1 \rightarrow[4 \rightarrow 6 \rightarrow 5 \rightarrow 9] \rightarrow 8 \rightarrow 10 \rightarrow[11 \rightarrow 12] \rightarrow[7 \rightarrow 3]
$$

\section{CONCLUSION}

A new solution methodology to the tool management problem is proposed in connection with the tool magazine arrangement and operations sequencing decisions. We utilize the benefits of tool sharing and loading duplicate tools due to a possible decrease in tooling and tool operating costs while maintaining the feasibility in terms of precedence, tool magazine capacity, tool availability and tool life covering constraints. So we not only improve the overall solution in terms of the total manufacturing cost, but also prevent any infeasibility that might occur at the system-level through a feedback mechanism. As a final point, an effective tool management is a major requirement for the implementation of an FMS, hence the CNC machine tools are stated by several authors. In the automated environments, sophisticated computerized decision making tools are needed for effective operation and control of the system. In this respect, this study can be considered as a part of the fully automated process planning system.

\section{REFERENCES}

1. J. S. Agapiou, Sequence of operations optimization in single-stage multi-functional systems, J. Manufact. Sys., 10, 194-208 (1991).

2. M. S. Akturk and S. Avci, Tool allocation and machining conditions optimization for CNC machines, Eur. J. Opl. Res., 94, 336-349 (1996).

3. K. Amoako-Gyampah, J. R. Meredith and A. Raturi, A comparison of tool management strategies and part selection rules for a flexible manufacturing system, Int. J. Prod. Res., 30, 733-748 (1992).

4. J. F. Bard, A heuristic for minimizing the number of tool switches on a flexible machine, IEE Trans., 20, 382-391 (1988).

5. Y. Crama, A. W. J. Kolen, A. G. Oerlemans and F. C. R. Spieksma, Minimizing the number of tool switches on a flexible machine, Int. J. Flexible Manufact. Syst., 6, 33-54 (1994).

6. A. E. Gray, A. Seidmann and K. E. Stecke, A synthesis of decision models for tool management in automated manufacturing, Mgmt Sci., 39, 549-567 (1993).

7. A. S. Kiran and R. J. Krason, Automated tooling in a flexible manufacturing system, Ind. Engr., $20,52-57$ (1988).

8. P. Kouvelis, An optimal tool selection procedure for the initial design phase of a flexible manufacturing system, Eur. J. Opl. Res., 55, 201-210 (1991).

9. S. C. Sarin and C. S. Chen, The machine loading and tool allocation problem in a flexible manufacturing system, Int. J. Prod. Res., 25, 1081-1094 (1987).

10. M. S. Sodhi, R. G. Askin and S. Sen, Multiperiod tool and production assignment in flexible manufacturing systems, Int. J. Prod. Res., 32, 1281-1294 (1994).

11. R. Suri and C. K. Whitney, Decision support requirements in flexible manufacturing, J. Manufact. Sys., 3, 61-69 (1984).

12. C. S. Tang and E. V. Denardo, Models arising from a flexible manufacturing machine, part I: minimization of the number of tool switches, Opns Res., 36, 767-777 (1988). 